Revue musicale OICRM

revue musicale oicrm

\title{
Écrits de compositeurs, médias et publics dans les années 1930. À propos des échanges sur le public de l'opéra entre Max d'Ollone et Charles Koechlin
}

\section{Michel Duchesneau}

Volume 7, numéro 1, 2020

URI : https://id.erudit.org/iderudit/1069477ar

DOI : https://doi.org/10.7202/1069477ar

Aller au sommaire du numéro

Éditeur(s)

Observatoire interdisciplinaire de création et recherche en musique (OICRM)

ISSN

2368-7061 (numérique)

Découvrir la revue

Citer cet article

Duchesneau, M. (2020). Écrits de compositeurs, médias et publics dans les années 1930. À propos des échanges sur le public de l'opéra entre Max d'Ollone et Charles Koechlin. Revue musicale OICRM, 7(1), 156-167.

https://doi.org/10.7202/1069477ar
Résumé de l'article

En explorant les échanges entre deux compositeurs, Max d'Ollone et Charles Koechlin dans Le Ménestrel et Le Monde musical en 1932, nous interrogerons leur conception du public de la musique afin de mieux comprendre la manière dont ils défendent une position artistique, voire esthétique. D’ollone soulève la question à savoir si les compositeurs doivent prendre en compte les goûts du public pour avoir du succès dans un contexte de crise du répertoire à l'opéra. Il n'en faut pas plus pour que Koechlin réagisse et propose une singulière catégorisation des publics qui tient à la fois compte des conditions sociales et des sensibilités de l'écoute. En se servant de leur pouvoir " médiatique » par l'entremise de revues musicales réputées, ils ne cherchent pas moins à influencer les points de vue, notamment en matière de goûts musicaux. L'intérêt de cet échange ne se limite pas au duel des compositeurs et des revues, car le directeur de l'Opéra-Comique, Georges Ricou, se mêle à la réflexion par l'intermédiaire du musicologue Paul Bertrand, chroniqueur dans Le Ménestrel. 


\title{
Écrits de compositeurs, médias et publics dans les années 1930. À propos des échanges sur le public de l'opéra entre Max d'Ollone et Charles Koechlin
}

\section{Michel Duchesneau}

\section{Résumé}

En explorant les échanges entre deux compositeurs, Max d'Ollone et Charles Koechlin dans Le Ménestrel et Le Monde musical en 1932, nous interrogerons leur conception du public de la musique afin de mieux comprendre la manière dont ils défendent une position artistique, voire esthétique. D'Ollone soulève la question à savoir si les compositeurs doivent prendre en compte les goûts du public pour avoir du succès dans un contexte de crise du répertoire à l'opéra. Il n'en faut pas plus pour que Koechlin réagisse et propose une singulière catégorisation des publics qui tient à la fois compte des conditions sociales et des sensibilités de l'écoute. En se servant de leur pouvoir " médiatique " par l'entremise de revues musicales réputées, ils ne cherchent pas moins à influencer les points de vue, notamment en matière de goûts musicaux. L'intérêt de cet échange ne se limite pas au duel des compositeurs et des revues, car le directeur de l'Opéra-Comique, Georges Ricou, se mêle à la réflexion par l'intermédiaire du musicologue Paul Bertrand, chroniqueur dans Le Ménestrel.

Mots clés : composition; goût musical; Charles Koechlin ; Max d'Ollone ; public de l'opéra.

\begin{abstract}
By exploring the exchanges between two composers, Max d'Ollone and Charles Koechlin in Le Ménestrel and Le Monde musical in 1932, we will question their conception of the public of music in order to better understand the way in which they defend an artistic, even aesthetic position. D'Ollone raised the question of whether composers must take into account the tastes of the public in order to be successful in a context of crisis in the operatic repertoire. Koechlin's response was to propose a singular categorization of audiences that took into account both social conditions and listening sensitivities. By using their "media" power through well-known music magazines, they seek no less to influence points of view, particularly in terms of musical taste. The interest of this exchange is not limited to the duel between composers and magazines, as the director of the Opéra-Comique, Georges Ricou, gets involved in the reflection through musicologist Paul Bertrand, columnist in Le Ménestrel.
\end{abstract}

Keywords: composition; Charles Koechlin; musical taste; Max d'Ollone; opera audience. 
Le rapport au public est certainement l'une des questions les plus importantes lorsqu'il s'agit de définir la place qu'occupent les compositeurs dans l'espace médiatique. C'est aussi par le filtre $d u$ "public » que se discutent de nombreuses questions d'ordre esthétique, même si bien des compositeurs ont toujours déclaré ne pas écrire pour un public en particulier. Il n'en reste pas moins que l'œuvre musicale doit une part de son existence à la présence d'un public en salle, d'un auditoire radiophonique, voire de discophiles. Les musiciens s'expriment depuis longtemps sur le sujet. Si les mémoires et les correspondances permettent de retracer le discours intime des compositeurs sur les publics, c'est dans la presse, par l'intermédiaire des entrevues mais aussi et surtout des articles de fond que l'on a accès aux prises de position les plus développées quant au rapport idéalisé ou concret que les compositeurs entretiennent avec le public. Entre la deuxième moitié du XIX ${ }^{e}$ siècle et les dernières décennies $\mathrm{du} \mathrm{xx}^{\mathrm{e}}$ siècle, la presse offre ainsi aux compositeurs un lieu médiatique idéal pour s'exprimer et entrer en contact avec les lecteurs qu'ils souhaitent convaincre de leurs idées, de leurs principes esthétiques et éventuellement de l'intérêt de leurs propres œuvres.

La réflexion que nous proposons ici s'appuie sur une étude de cas, celui d'un échange entre deux compositeurs français, Max d'Ollone (1875-1959) et Charles Koechlin (1867-1950), un échange né autour d'une « crise » au sein du milieu musical français des années 1930, cristallisée autour de l'Opéra-Comique qui croule alors sous d'importants déficits et peine à trouver son public. Outre une situation financière réellement difficile dont les causes sont multiples, parmi lesquelles il faut prendre en considération la crise économique générale qui frappe alors la France, la question du rapport du public avec les institutions et le répertoire semble aussi compter. Si dans un premier article publié dans la Revue des deux mondes en juillet 1931, le codirecteur de l'Opéra-Comique, Georges Ricou ${ }^{1}$ met en cause spécifiquement le nouveau mode de vie et la concurrence du disque, de la radio et du cinéma, dans un second article d'août 1932, il insiste plus précisément sur le fait que la scène lyrique française souffre aussi d'une désaffection de la part du public. Ce second problème s'appuie sur le constat qu'

obéissant [...] à un désir d'évolution, à une volonté de renouvellement et de progrès, les compositeurs chargés d'alimenter par leurs œuvres le répertoire de ces deux théâtres abandonnent de plus en plus les formes traditionnelles. Moins soucieux de facilité et de charme que de science et d'érudition technique, ils s'efforcent d'accroître le domaine de la musique pure [...] À ce jeu, le public, peu à peu, s'est écarté des œuvres nouvelles. Il a perdu le goût de les connaître, la curiosité préalable, climat nécessaire à l'épanouissement fécond et durable de la production musicale (Ricou 1932, p. 622-623).

L'échange qui va suivre entre d'Ollone et Koechlin s'appuie sur la seconde intervention de Ricou et l'écho qu'elle connaît dans Le Ménestrel sous la plume

1 Selon la notice bibliographique de la Bibliothèque nationale de France, Georges Ricou (1880-19?), homme de lettres et metteur en scène, fut codirecteur (avec Louis Masson) de l'Opéra-comique de 1925 à 1931. 
de Paul Bertrand ${ }^{2}$. La réflexion des deux compositeurs permet non seulement de dégager les grandes idées à propos du " problème » du public de la musique savante à l'époque, mais il nous permet aussi de réfléchir sur ce que représente cette prise de parole publique de la part de musiciens. Bien qu'ils soient tous les deux connus pour leur engagement envers le milieu que ce soit d'Ollone, qui a occupé plusieurs fonctions dans l'enseignement et l'administration de la musique, ou Koechlin, qui prend la parole très régulièrement dans la presse musicale et généraliste pour soutenir la cause de la musique, les échanges entre les deux compositeurs dont il sera question ici témoignent d'une conscience peut-être plus aiguë de leur rôle social et par le fait même, de leurs devoirs et prérogatives.

La question du public est délicate à aborder dans le contexte de la prise de parole des artistes, car au-delà des habituelles récriminations quant au conservatisme des auditeurs, les compositeurs ont fort à faire lorsqu'il s'agit de définir leur propre relation avec le public. Sur quoi se fonde cette relation? Pour une majorité, du moins à l'époque, le public de la musique est plus ou moins homogène et divisé en deux : celui qui fréquente les concerts dans lesquels leurs œuvres sont jouées et celui qui ne fréquente pas ces concerts ${ }^{3}$. Le non-public n'est pas à l'époque un concept bien établi, si ce n'est par l'idée qu'il existe une part de la population qui ne possède pas le bagage culturel ou les moyens nécessaires pour partager l'amour de la " grande musique ». Si l'on se fie aux commentaires de Koechlin et d'Ollone, la relation s'établit essentiellement sur une série d'a priori que l'expérience tend à confirmer dans la mesure où la rigidité du cadre social du concert maintient ces a priori.

Il faut aussi se poser la question de la manière dont les compositeurs utilisent la presse au cours de la première moitié du $\mathrm{xx}^{\mathrm{e}}$ siècle pour entrer en dialogue avec «leur» public et éventuellement convaincre un " autre " public de l'intérêt de leur démarche. Comment s'articulent leurs idées sur les auditeurs et la nature du support médiatique qu'ils utilisent dans l'optique, à peine dissimulée, de défendre une position artistique, voire esthétique de leur part ? Cette question est d'autant plus importante que les revues dans lesquelles on trouve la majorité des interventions des compositeurs sont des revues d'art, souvent spécialisées dans le domaine musical. Le lectorat n'est-il pas alors un public déjà acquis ? À la mélomanie, du moins?

2 À la suite de la parution de l'article de Ricou dans la Revue des deux mondes, Paul Bertrand, dont on ne connaît que peu de choses si ce n'est qu'il travaille chez Heugel comme éditeur et qu'il tient de nombreuses chroniques dans Le Ménestrel, publie une synthèse intitulée "L'Avenir du Théâtre » dans Le Ménestrel du 12 août 1932. L'année précédente, Bertrand avait déjà fait une large place à la question de la crise des scènes lyrique en publiant «La Crise des Théâtres lyriques subventionnés » dans Le Ménestrel des 12 et 19 juin 1931. Ricou enchaînait en publiant en juillet 1931 "La crise de l'Opéra-comique et la crise générale du théâtre » dans la Revue des deux mondes et Bertrand poursuivait toujours dans Le Ménestrel avec un article « La Crise de l'Opéra-Comique » en trois parties, les 17, 24 et 31 juillet 1931.

3 Mais ce n'est pas parce que le public est absent du concert qu'il n'est pas connaisseur et qu'il ne fréquente pas les œuvres contemporaines. Dans les années 1930, disque et radio jouent un rôle très important dans la diffusion musicale et même si les compositeurs n'y trouvent pas toujours leur compte, ces moyens de diffusion contribuent à la circulation de leurs œuvres à des degrés divers cependant. 
La question de la conquête de nouveaux publics par leurs interventions médiatiques peut être résumée à l'idée de prêcher pour les convertis, on peut cependant se demander comment ils se servent de leur pouvoir " médiatique » pour influencer les points de vue, notamment en matière de goûts musicaux, car il y a là, malgré tout, un enjeu de taille. Le succès public n'est pas seulement celui du nombre, mais aussi celui de l'estime. Les concerts où furent créées plusieurs œuvres célèbres au tournant du $\mathrm{XX}^{\mathrm{e}}$ siècle ne réunissaient assurément pas des foules immenses ${ }^{4}$. Le succès est d'abord et avant tout celui gagné auprès du public qui « fait » la réputation du musicien et qui lui permet de s'inscrire dans l'histoire. Cette histoire est le produit du travail d'une partie de ce public que nous oserons qualifier d'élite. Il discute et critique les œuvres, mais il est aussi celui que Koechlin qualifiera de "snob », c'est-à-dire ce public dont les habitudes culturelles sont davantage le fait de conventions sociales que d'une réelle passion pour la musique, et cela même s'il s'agit en partie d'une simple assiduité basée sur une sociabilité à entretenir.

LE PRÉ AU DUEL

Notre étude s'appuie donc sur les échanges entre d'Ollone et Koechlin publié dans Le Ménestrel et Le Monde musical en 1932.

D'Ollone a connu une carrière brillante de compositeur (prix de Rome en 1897), de professeur - d'abord à l'École normale de musique puis au Conservatoire - et d'administrateur de la musique, même si sa participation à la section musicale du Groupe collaboration pendant la Seconde Guerre mondiale entacha cette carrière. Considéré comme un grand musicien par la majorité de ses contemporains, Koechlin fut d'ailleurs l'un de ceux qui le défendirent lors des accusations de collaboration qu'il eut à subir. D'Ollone fut acquitté compte tenu de ses antécédents. L'intervention de Koechlin n'est pas étonnante, car les deux hommes se connaissent très bien. Ils ont été camarades dans les classes d'André Gédalge et de Jules Massenet dans les années 1893-18955 . D'Ollone écrivit très régulièrement pour l'opéra et plusieurs de ses œuvres furent créées par les scènes nationales dont L'arlequin à 1'Opéra de Paris en 1924, une partition qui connut, semble-t-il, le succès et des critiques très favorables. Outre ses interventions dans la presse, notons qu'il est l'auteur de certains ouvrages qui méritent d'être cités, notamment Langage musical publié en deux volumes en 1952 ainsi que Le théâtre lyrique et le public publié en 1955 et qui reprendra certains arguments des articles de 1932.

De huit ans son aîné, Koechlin connaît aussi une carrière brillante, mais très différente, car s'écartant du parcours institutionnel - pas de prix de Rome, pas de

$4 \quad$ Les concerts de musique de chambre de la Société nationale de musique et de la Société musicale indépendante ne devaient pas réunir plus de quelques centaines d'auditeurs. Les concerts avec orchestre offraient de meilleures perspectives en matière d'affluence.

5 Dans ses souvenirs, d'Ollone cite Koechlin comme l'un de ses camarades de classe chez Massenet (Ollone 1948, p. 737). Koechlin commentera abondamment l'œuvre de d'Ollone tout au long de sa vie, notamment les œuvres pour la scène. Parmi les premiers textes publiés, relevons un compte rendu paru dans Le Mercure de France sur l'exécution du ballet Bacchus et Silène de d'Ollone donné aux Arènes de Bézier. 
postes officiels, pas d'œuvres lyriques créées sur les scènes nationales. Son catalogue se caractérise par un nombre considérable d'œuvres instrumentales de musique de chambre et symphoniques dont l'ampleur, pour ces dernières, atteint dans certain cas des dimensions considérables, des ouvrages théoriques dont l'importance reste d'actualité, comme son Traité d'orchestration en quatre volumes ${ }^{6}$ et un nombre appréciable de contributions dans la presse musicale qui abordent une foule de sujets.

Les échanges entre les deux compositeurs donne lieu à huit textes qui paraîtront dans Le Ménestrel et Le Monde musical entre le 29 juillet et le 30 décembre 1932. D’Ollone publie ses articles dans Le Ménestrel alors que ceux de Koechlin le sont dans Le Monde musical, revue concurrente, mais dont les allégeances ne sont pas si opposées, car les réseaux de sympathie musicale et politique sont difficiles à démêler et ne permettent pas d'identifier très clairement la position esthético-politique de chacune de ses revues.

Que doit-on voir alors dans le fait que les compositeurs n'écrivent pas dans la même revue ? Le Ménestrel est alors dirigé par Jacques Heugel, éditeur bien connu, dont la revue fondée en 1833 possède une solide réputation et tient une chronique de la vie musicale et lyrique très suivie, tout comme de l'activité du Conservatoire. La revue est aussi un support indispensable pour la maison d'édition qui y fait une promotion constante des partitions éditées. Dans le numéro de juillet 1932, on repère la réduction pour piano de la partition du ballet Le temple abandonné et une mélodie Berceuse persane de d'Ollone, dans la liste des œuvres éditées peu de temps auparavant. Si l'on jette un coup d'œil au catalogue d'Heugel, on y trouve plusieurs œuvres de d'Ollone, entre autres 1'opéra L'arlequin et l'opéra-comique Georges Dandin (1930). Pour Le Ménestrel, donner la parole à un compositeur dont la maison d'édition finançant la revue publie les œuvres ne peut être que bénéfique. Lorsque Le Ménestrel publie le premier article de d'Ollone, la rédaction ne manque pas d'indiquer combien cette prise de parole est importante :

À maintes reprises, Le Ménestrel a montré que la crise actuelle de l'art lyrique comportait, parmi beaucoup d'autres, une cause fondamentale d'ordre esthétique, à laquelle il appartient surtout aux compositeurs de remédier.

L'article ferme et libre que l'on va lire a le grand mérite de poser la question dans toute son ampleur. Il emprunte à la personnalité de son auteur une autorité particulière (dans Koechlin 2009, p. 187).

Il s'agit donc pour Le Ménestrel non plus de communiquer de manière journalistique des informations sur l'état de l'Opéra-Comique, comme il l'a fait précédemment, entre autres en publiant des textes de Paul Bertrand et de Jean Dupérier entre 1921 et 1931 (voir Bertrand 1921 et Dupérier 1930) à propos de la crise presque permanente que vivent les théâtres lyriques en France, mais bien d'aborder la problématique d'un point de vue "esthétique", ce que seul un compositeur peut entreprendre dans la mesure où sa prise de parole est légitimée par sa position cardinale dans la chaîne de coopération de la création musicale. À quoi sert une telle intervention pour Le Ménestrel ? Outre le prestige du compositeur qui rejaillit sur la revue, du moins pour les lecteurs qui

6 À propos du Traité d'orchestration, voir Duchesneau 2013. 
connaissent le compositeur, il s'agit d'offrir au lectorat plus large de la revue, une réflexion de «l'intérieur ». En donnant la parole à d'Ollone, professeur au Conservatoire et musicien adoubé par les institutions musicales de l'État, Le Ménestrel renforce sa position de média qui articule vie musicale, information et opinions. Les opinions du compositeur sont d'autant plus précieuses pour la revue qu'il s'agit d'adopter le point de vue du public. Dans l'esprit de l'éditeur, ce public se réclame d'une approche " populaire » de la création musicale qui s'écarte délibérément de celle d'une élite avant-gardiste. C'est un discours médiatique qui ne peut qu'emporter l'adhésion d'un lectorat beaucoup plus large que celui représenté par cette élite au contour cependant flou. Dans un tel environnement éditorial, Koechlin, étroitement associé à une modernité résolue et l'un des animateurs de la Société musicale indépendante, peut-il répondre à d'Ollone dans les pages du Ménestrel ? Rien ne permet de le confirmer, sachant que Koechlin aura déjà écrit pour Le Ménestrel dans les années 1920.

Koechlin réagit aux propos de d'Ollone dans Le Monde musical, dirigé par Auguste Mangeot, le fils du facteur de pianos Édouard Mangeot. Auguste est 1'un des fondateurs de l'École normale de musique de Paris. La revue suit les activités de l'École normale, mais offre aussi un panorama musical très large, en adoptant une approche héritée de la défunte revue Musica, c'est-à-dire en exploitant la photographie. Elle est également moins orientée que Le Ménestrel, notamment parce que la revue, malgré sa relation avec l'École normale, fait état de manière beaucoup plus étendue de la vie musicale hors de la zone d'influence du Conservatoire et de la scène lyrique et accorde une place substantielle aux concerts instrumentaux. La revue accueille régulièrement les textes de Koechlin, mais cette participation n'est pas exclusive : non seulement le compositeur publiera dans Le Ménestrel entre 1921 et 1936, mais on trouvera des articles du compositeur publiés dans les Tablettes de la Schola, Le Courrier musical, La Pensée, Contrepoints et La Revue musicale, cette dernière remportant la palme $\mathrm{du}$ plus grand nombre de contributions du compositeur entre les deux guerres.

Le premier texte de d'Ollone porte le titre "Les musiciens et le public » et aborde la question du " désaccord foncier qui s'est établi peu à peu entre les musiciens et le public » (dans Koechlin 2009, p. 187). D'Ollone soulève la question de savoir si les compositeurs doivent prendre en compte les goûts du public pour avoir du succès dans un contexte de crise du répertoire à l'opéra. Il pose au lectorat du Ménestrel une question directe : le désengagement des institutions envers les œuvres contemporaines serait-il le fait des musiciens eux-mêmes qui jadis écrivaient pour le public et qui « en sont arrivés à écrire "contre" lui ?» (ibid.). Le compositeur s'engage dans une manœuvre délicate, car il oppose les œuvres issues de l'intellect, qu'il considère alors difficilement accessibles au public, et les œuvres nées de l'instinct, d'un don et d'une sensibilité « naturelle ». Il précise :

Du moment que la musique n'était plus l'apanage de l'instinct, du don spécial, d'une sensibilité singulière, d'une oreille affinée, d'une écriture particulière, compositeurs et amateurs devaient s'incliner devant les intelligences supérieures qui, seules, pouvaient en mesurer la valeur spirituelle, c'est-à-dire devant les littérateurs... Cela 
donna un poids énorme et nouveau à la critique ; cela permit à tout mondain lettré de juger des choses de la musique (dans Koechlin 2009, p. 190).

L'enjeu est donc celui de la "spécialisation » de l'écriture musicale, spécialisation qui écarte bon nombre d'auditeurs qui ne possèdent pas ou plus les codes pour déchiffrer la musique qui leur est proposée. Wagner, la Schola, Debussy sont tour à tour convoqués pour expliquer l'élitisation de la musique et le développement déraisonnable du drame musical, "genre bâtard qui a fait perdre au public le chemin » (ibid., p. 192) de la salle d'Opéra.

Le verdict de d'Ollone est clair :

Jadis un amateur n'éprouvait pas seulement, au théâtre et au concert, un plaisir d'oreille et une émotion d'ordre sentimental : il éprouvait la jouissance de comprendre l'art musical. Si comprendre c'est reconnaître, le public le plus cultivé en est arrivé graduellement à ne plus comprendre la musique - même celle dont il subit l'attrait - parce qu'on a peu à peu brisé les moules et que chacun cherche à se créer son vocabulaire et sa syntaxe d'où la confusion générale, où les musiciens euxmêmes ne se retrouvent plus (ibid., p. 191).

Cette prise de position suscite chez Koechlin une vive réaction articulée sur trois axes de force : 1) la défense du drame symphonique (sa réponse est titrée "Pour le drame symphonique » (ibid., p. 196) ; 2) la critique de la vision téléologique de l'histoire du répertoire lyrique qui ferait en sorte que le public du XIX siècle se " ruait aux nouveautés » (ibid., p. 197) et que le public contemporain ne s'y intéresserait plus, faute d'en comprendre la musique ; et 3)l'idée que le public n'est pas le groupe homogène pensé comme tel par d'Ollone.

Koechlin débute sa réponse en résumant à sa manière l'article de son collègue compositeur. Il identifie trois saillances :

$1^{\circ}$ / Les théâtres de musique, en raison des frais énormes qu'ils comportent, ne peuvent vivre avec une clientèle restreinte.

$2^{\circ}$ / Il est nécessaire, à tout point de vue, de donner de la musique à la foule.

$3^{\circ}$ / Celle-ci, maintenant comme toujours, réclame de la musique qui vienne [sic] du cœur et qui chante (ibid., p. 196).

Il faut noter le terme employé par Koechlin : il ne parle pas de public, mais de foule. La nuance fait intervenir la notion de ce que nous pourrions appeler aujourd'hui grand public, lequel se distinguerait d'un public d'élite. C'est pour ce dernier, relève Koechlin, que les compositeurs, d'après d'Ollone, écrivent désormais, alors que le public en général, la « foule » chez Koechlin, voudrait plutôt de «belles voix [et] de bons chanteurs » (ibid.).

D'une part, Koechlin interroge l'idée d'un public monolithique qui ne ferait qu'un et dont les goûts musicaux seraient unanimement partagés et, d'autre part, il lui semble difficile d'affirmer qu'autrefois, le public adoptait immédiatement les œuvres nouvelles. Il faut alors saisir la nuance qu'apporte ici Koechlin. Pour cela, il faut être attentif au mot mis en italiques pour qualifier la musique dont il est question : "nouvelle ».

Ce qu'il sous-entend, est une idée assez simple : ce n'est pas parce qu'une œuvre est jouée pour la première fois qu'elle est forcément " nouvelle ». Koechlin précise : 
"En réalité - et ceci ne date d'aujourd'hui, ni d'hier - toute œuvre nouvelle a eu, a, aura plus ou moins de mal pour être comprise et percer $^{7}$ " (Koechlin 2009, p. 197). La nouveauté d'une œuvre se définit à partir de ses caractéristiques intrinsèques qui la distinguent des œuvres du répertoire et de la production contemporaine conservatrice. L'analyse de Koechlin s'appuie sur l'idée que la nouveauté a toujours été source de conflit entre les créateurs et le public, et que, par conséquent, il est faux de prétendre que l'ordre contemporain soit foncièrement différent de celui du passé, d'autant que le public n'a jamais été univoque.

Considérant le public comme une entité sociale, Koechlin lui accole un double principe de variété : l'œuvre musicale est inévitablement variée et le public est en lui-même aussi varié. L'adéquation doit alors se faire entre une musique en particulier et un public en particulier qui se définit par le fait qu'il y trouve l'expression de ses goûts musicaux, qui de plus ne sont pas forcément figés :

Cette « musique vocale sous toutes ses formes » que semble demander le public au théâtre musical, elle peut donc affecter des aspects divers, étant fonction des époques et des natures. Il n'y a pas un type modèle de «mélodie chantante » : il n'y a pas non plus un seul goût du public exigeant cette mélodie-standard; et nous devons admettre pour évident que le public lui-même évolue, d'un siècle, voire d'une génération à l'autre (ibid., p. 198).

L'occasion est trop belle pour Koechlin de ne pas élaborer une catégorisation éminemment personnelle des publics de l'opéra qui, bien qu'elle puisse faire sourire de nos jours, n'est cependant pas si éloignée de certaines autres catégorisations qui naissent à l'époque. Nous pensons évidemment à celle d'Adorno, sans pour autant vouloir faire de la réflexion de Koechlin ce qu'elle n'est pas, c'est-à-dire une sociologie des publics de la musique. La classification de Koechlin s'appuie sur les genres musicaux, ce qui n'est pas sans intérêt, car au fond, ces genres impliquent aussi une organisation de la vie de concert autour de l'Opéra-Comique et de l'Opéra :

Il y a, d'abord, le public d'opérette, pour qui trop raffinée était L'Étoile, trop sérieux et trop "wagnérien » le Sigurd de Reyer (si mélodique pourtant ; et dont je regrette de ne plus voir le nom sur l'affiche où s'étale celui de Thaïs). Public qui songe avant tout à s'amuser, tient en mépris «le classique », admet le jazz pour sa gaîté rythmique et se plaît aux danses comme aux chansons les plus niaisement plates. [...]

Un autre public - celui que vise $\mathrm{M}$. d'Ollone - soutient le répertoire des théâtres lyriques subventionnés ; s'il admet à l'occasion des chefs-d'œuvre (Faust, Carmen), il a fait la fortune de Mignon, par un mystère encore non éclairci ; son dangereux éclectisme accepte, pêle-mêle, le meilleur comme le pire de Massenet, et son culte pour la musique italienne se manifeste par un amour sans bornes pour Madame Butterfly, La Bohème, Paillasse (hélas !), la Tosca (holà !).

$7 \quad$ Koechlin ajoute en note de bas de page à son article : « Mais ce qui n'est point compris n'est pas forcément bon, et ne point percer n'est pas un critérium certain de la beauté. On sait plus d'un médiocre qui ne « percera » jamais : pas assez mauvais pour plaire à la masse, pas assez bon pour l'élite. » 
Enfin, il existe un troisième public. Et l'on aura beau railler les pauvres snobs qui tiennent à l'honneur de faire partie de l'«élite »: je ne [peux qu'admettre] que ce troisième public, même mélangé de snobs, a toutes mes sympathies, ayant soutenu Le Roi malgrélui, Le Rêve, Messidor, Guercour, Fervaal, Boris Godounov, Pelléas, Pénélope, Padmâvati. À coup sûr, il est moins nombreux ; beaucoup moins ! Mais fidèle et compréhensif, c'est lui qui sauve la musique (Koechlin 2009, p. 199).

À la suite du premier échange, les compositeurs continueront la discussion. L'objectif pour d'Ollone est clairement de contribuer à éclairer les lecteurs des deux revues à propos de la question fondamentale du rapport entre le compositeur et son public à travers son œuvre ${ }^{8}$. Sans vouloir décortiquer ces échanges dans le cadre de cette courte étude de cas, on peut résumer la suite du débat par une idée forte de d'Ollone exprimée dans sa réponse à Koechlin en décembre 1932 : la réflexion de Koechlin est pour lui d'ordre esthétique, alors que la question est pratique, car « c'est le public qui fait vivre le théâtre » (ibid., p. 215).

L'incompréhension des œuvres (leur insuccès lors des premières) serait surtout le fait des publics d'élite et pas de la «foule " dont l'engagement envers une œuvre est plus important qu'on l'imagine. D'Ollone cite le cas de Samson et Dalila dont le succès est né de l'accueil du public de Rouen et de la "popularité de certains morceaux " (dans ibid., p. 216). Il termine son article par la défense du rapport instinctif à la musique :

Un éminent compositeur me dit un jour : "Je termine un drame lyrique. Mais je songe avec tristesse, en fignolant avec amour mille détails d'orchestration, que le public n'y prêtera pas attention ». Évidemment, cher Maître. Ni moi non plus... Assis à côté de la femme d'un autre éminent compositeur, je la vis avec étonnement applaudir une œuvre qui me semblait horrible : «Ce n'est sans doute pas très beau, me dit-elle, mais, au moins, ce n'est pas sentimental. [...] J'aime[rais] pourtant, ô Charles Kœchlin, être entraîné dans un monde meilleur, par l'adagio du Deuxième Quintette de Fauré... Mais je ne suis pas venu chercher ici l'initiation pythagoricienne. Chaque chose en son temps et lieu. Et Wagner ne voulait Parsifal qu'au temple de Bayreuth !

Art grossier, pour un public grossier ? Qui sait ! Né de l'instinct, s'adressant à l'instinct, au cœur, aux sens, aux nerfs, il atteint en l'être humain des couches profondes. "L'intelligence », disait Barrès, «quelle petite chose à la surface de notre être! Profondément nous sommes des êtres affectifs» (ibid., p. 221).

Les positions sont irrémédiablement irréconciliables, car lorsque Koechlin place l'intellect très haut dans la hiérarchie de l'expression musicale, sans nier l'apport de l'instinct, il défend une approche « intellectuelle » de la composition :

D'une façon plus générale, j'estime que l'artiste et l'intellectuel ne doivent pas être séparés. Tout « intellectuel » qui se consacre à l'art des sons pour le juger, doit être

8 Dans sa réponse d'Ollone explique aux lecteurs : « Ce qui me pousse à reprendre la plume, c'est que nous traitons là d'un sujet intéressant un assez grand nombre de gens, sujet actuel au premier chef, et que, le discutant en toute indépendance d'esprit, et aussi objectivement que possible, nous pourrions, de nos divergences même, faire naître quelque utile lueur » (dans Koechlin 2009, p. 215). 
capable de sentir vivement, de comprendre ce que signifie une musique, même " pure " (je ne dis pas de traduire cela par des mots). Tout artiste, d'autre part, gagne à être aussi un intellectuel, c'est-à-dire à savoir réfléchir (ce qui ne signifie point : avoir des thèses $a$ priori $),-$ à savoir construire ses ouvrages avec ordre : ordre qui ne suffit pas, mais qui est nécessaire, et d'ailleurs réalisable de cent manières (Koechlin 2009, p. 211).

\section{EN GUISE DE CONCLUSION PROVISOIRE : CONVAINCRE QUI ?}

Les échanges entre d'Ollone et Koechlin, à travers le prisme de la crise de l'opéra, fait apparaître la fracture entre compositeurs et public, fracture qui se décline en une opposition féroce entre deux idées antagonistes que résume Ricou : la musique comme forme d'expression intellectuelle et la musique comme forme d'expression instinctive habitée par le charme et la séduction (dans Bertrand 1932, p. 337).

En écrivant dans Le Ménestrel et Le Monde musical, les compositeurs prennent appui sur un lectorat engagé, ne serait-ce que parce qu'il s'agit d'une presse spécialisée essentiellement soutenue par des abonnés, mélomanes plus ou moins avertis. On peut donc considérer que la différenciation des profils des lecteurs en fonction du média est réduite à peu de chose. On peut cependant soupçonner une allégeance plus ou moins grande aux institutions que sont le Conservatoire, l'École normale, voire la Schola Cantorum. Il ne s'agit donc pas pour les compositeurs de mobiliser un camp plutôt qu'un autre, mais de plutôt défendre une conception esthétique et par conséquent un rapport très personnel à la composition comme action qui inscrit le créateur au sein de la société (ou pas). Si pour d'Ollone l'enjeu du public de l'opéra a une réelle importance, sa position de professeur au Conservatoire et directeur du Conservatoire américain de Fontainebleau lui permet de garder une certaine distance. Pour Koechlin, il en va différemment, dans la mesure où sa musique trouve difficilement place au concert alors que la reconnaissance du public est essentielle lorsqu'il s'agit de convaincre un organisateur de concert ou un éditeur de diffuser sa musique.

Partant d'un problème institutionnel, celui du répertoire de l'Opéra et de l'adhésion ou pas du public à ce répertoire, problème pratique de gestion artistique qui se résume à une question d'impératifs financiers, les compositeurs plaident pour une prise en compte esthétique de ce que doit être une œuvre musicale, prise en compte qui d'après d'Ollone ne peut échapper au contexte de création, mais surtout, ne doit pas faire l'impasse du goût du public. Pour d'Ollone, il faut écrire " pour le public ", puisque c'est lui qui fait vivre les théâtres. Pour Koechlin, ce n'est pas acceptable, car cela remet en cause l'indépendance artistique :

Ne pas obéir au public, même en matière de théâtre lyrique, que cette obéissance ne soit conforme à votre tendance naturelle. Ce qui tuerait notre musique (je dis tuerait, car il ne me semble pas que rien ne la tue pour l'instant, sauf les difficultés matérielles de la vie), ce serait d'obéir, précisément, à ces goûts de la masse, sans y être conduit par un goût pareil et irrésistible. Il faut, au contraire, éduquer le public, le guider. Tout cela, direz-vous, c'est très joli, mais comment faire ? Et l'on m'attend sans doute à la réalisation de ces desiderata. On m'objectera que $\mathrm{M}$. d'Ollone, lui, suggère une solution pratique alors qu'idéologue, utopiste, épris de songe et de chimère, je n'en donne aucune. 
J'avoue n'avoir pas pensé, tout d'abord, à cette « question pratique », estimant qu'il faut toujours poser comme possibles ces résultats qu'on demande à la vie. Supposer, a priori, réalisable et non absurde une découverte ou la solution d'un problème : puis, chercher comment y parvenir.

Si, de maintenir au répertoire d'autres œuvres que celles réclamées par la foule, il doit résulter un déficit pour les théâtres lyriques, et qu'ainsi ce ne soit plus qu'une question d'argent (si aiguë soit-elle), ne peut-on la résoudre autrement que par la suppression de ces œuvres moins favorisées du « suffrage universel »? (Koechlin 2009, p. 209)

Les lecteurs du Ménestrel et du Monde musical vont à l'Opéra et à l'Opéra-Comique. Ce sont aussi les auditeurs de la Société nationale, tout comme du tout nouvel Orchestre de Paris. Ils lisent aussi La Revue musicale et ses articles de fond comme les chroniques discographiques de son directeur Henry Prunières. La « foule » cependant ne lit probablement pas Le Ménestrel ni Le Monde musical. Le public dans les faits est celui du milieu musical : les musiciens d'abord, les directeurs, les critiques qui font l'opinion et les mélomanes formant une bonne partie du public d'élite. C'est fondamentalement ceux et celles qui font le monde du concert. Pour les deux compositeurs, il s'agit donc à la fois d'affirmer ce qu'ils sont en tant que créateurs auprès de ce lectorat choisi, mais aussi de confirmer une position dans le champ musical, associée à un rapport plus ou moins étroit avec les publics auxquels s'identifient les lecteurs, en fonction de leurs goûts musicaux.

L'intérêt de ces échanges ne se limite pas au duel des compositeurs et des revues, car Bertrand, chroniqueur au Ménestrel, et Ricou, directeur de 1'Opéra-Comique, auront mis la table dès les mois de juin et juillet 1931dans leurs textes parus dans Le Ménestrel et La Revue des deux mondes. Le corpus de textes constitué autour de cette question s'élargira constamment. Koechlin reprendra le thème dans un texte intitulé "Art et public " publié à nouveau dans Le Monde musical en octobre 1937 (ibid., p. 307-317). Dans ce texte, Koechlin nous éclaire sur l'enjeu médiatique qui consiste à occuper l'espace de médiation que représente alors les pages des revues. Sans pour autant abandonner le principe de l'autonomie de l'œuvre d'art, Koechlin adopte une position de passeur dont les explications renforcent la position de l'artiste " qui a le sens du rêve et de la beauté » et qui doit défendre cette position dans une société moderne qui le réduit progressivement au silence, car ses œuvres ne trouvent plus à être jouées et par conséquent entendues. Héritier d'un romantisme affirmé, le compositeur prendra la parole pour dénoncer une situation de marginalisation qu'il ne peut accepter, il conclura pour nous : "l'art est une lutte constante de l'artiste et du goût de son public! Une œuvre personnelle inquiète et déconcerte l'auditeur, comme toute chose nouvelle inquiète et déconcerte le commun des mortels » (ibid., p. 316). 


\section{BIBLIOGRAPHIE}

Bertrand, Paul (1921), «Musique pure et Musique dramatique », Le Ménestrel, 83e année, $\mathrm{n}^{\circ} 23$ (10 juin), p. 241-243, http://1mhsbd.oicrm.org/media/public/documents/ART-BEPa-1921-02.pdf ; n 24 (17 juin), p. 249-251, http://lmhsbd.oicrm.org/media/public/documents/ART-BEPa-1921-03.pdf, consultés le 5 janvier 2020 .

B[ertrand], P[aul] (1931a), «La Crise de 1'Opéra-Comique », Le Ménestrel, 93e année, n 29 (17 juillet), p. 319-320; $\mathrm{n}^{\circ} 30$ (24 juillet), p. 327-328; $\mathrm{n}^{\circ} 31$ (31 juillet), p. 335-338, https://gallica.bnf.fr/ ark:/12148/cb344939836/date/1931, consulté le 5 janvier 2020.

Bertrand, Paul (1931b), «La Crise des Théâtres lyriques subventionnés ", Le Ménestrel, 93e année, $\mathrm{n}^{\circ} 24$ (12 juin), p. 253-256, http://1mhsbd.oicrm.org/media/public/documents/ART-BEPa-1931-01. pdf ; n 25 (19 juin), p. 265-268, http://1mhsbd.oicrm.org/media/public/documents/ARTBEPa-1931-02.pdf, consultés le 5 janvier 2020.

Bertrand, Paul (1932), "L'Avenir du Théâtre », Le Ménestrel, 94e année, no 33 (12 août), p. 337-340, https://gallica.bnf.fr/ark:/12148/bpt6k5618289n.item, consulté le 5 janvier 2020.

Duchesneau, Michel (2013), "Raisonner l'orchestration. Introduction au Traité d'orchestration de Charles Koechlin ", Nicolas Donin et Laurent Feneyrou (dir.), Théorie et composition musicales au XX siècle, vol. 1, Lyon, Symétrie, p. 429-452.

Dupérier, Jean (1930), "À propos du Théâtre Lyrique », Le Ménestrel, 92e année, no 7 (14 février), p. 69-71, http://lmhsbd.oicrm.org/media/public/documents/ART-DUJa-1930-01.pdf, consulté le 5 janvier 2020.

Koechlin, Charles (2009), Musique et société, écrits présentés par Michel Duchesneau et annotés par Michel Duchesneau, Audrée Descheneaux et Danick Trottier, Sprimont, Mardaga.

Ollone, Max d' (1948), «Souvenirs d'un compositeur », Revue des deux mondes, vol. 83 (15 décembre), p. 729 738, https://www.revuedesdeuxmondes.fr/article-revue/souvenir-dun-compositeur/, consulté le 5 janvier 2020.

Ollone, Max d' (1952), Langage musical, Paris, Palatine.

Ollone, Max d' (1955), Le théâtre lyrique et le public, Paris, Palatine.

Ricou, Georges (1931), "La crise de l'Opéra-comique et la crise générale du théâtre ", Revue des deux mondes, juillet, p. 83-109.

Ricou, Georges (1932), «L'avenir du théâtre », Revue des deux mondes, $1^{\text {er }}$ août, p. 619-639. 